\title{
Czernowitz und die Bukowina
}

\author{
Martin A. Hainz
}

Czernowitz ist heute eine regional bedeutsame Ortschaft - Hauptstadt der Oblast Tscherniwzi und die traditionelle Hauptstadt der Bukowina im Karpatenvorland mit einer knappen Viertelmillion Einwohner und einem florierenden Markt, gelegen in der Westukraine; der Handel ergibt sich unter anderem aus der Nähe der rumänischen Grenze und aus der einstigen Zugehörigkeit zur UdSSR. Czernowitz war immer entweder der Vorposten einer Kultur ins Nichts (nach dem Maßstab der expandierenden Kultur) bzw. der ,Natur' oder der exponierte Ort, der Welten verband. Umso erstaunlicher ist die kulturelle Bedeutung, zu der es diese aus einer Garnison gewachsene Oase der Kultur brachte. Die Bukowina ist zunächst schütter besiedelt, verschiedene Dokumente und Zeugnisse, darunter auch literarische wie Moses Rosenkranz' Memoiren, machen deutlich, dass diese Region nur bäuerliche Besiedelung kennt, mit Häusern mit Lehmböden noch zu einer Zeit, in der Czernowitz bereits städtische Züge hat und der Landbevölkerung als „beutereiches Dickicht“ (Rosenkranz 2001, 212) erscheint (vgl. Mittelmann 2001, 29).

Czernowitz ist wesentlich der expansive Vorstoß Habsburgs, sieht man von der weniger ergiebigen Vorgeschichte ab: Zunächst als befestigte Siedlung am linken Ufer des Pruths aus der Zeit der Kiewer Rus unter Rurikiden-Fürst Jaroslaw Osmomysl gegründet, der zwischen 1153 und 1187 regierte, ist Czernowitz erst 1408 urkundlich erwähnt, zuvor wird in Legenden die Stadt Tschern (Schwarze Stadt) genannt. Die Spuren der ersten Kultivierung sind marginal, die Festung wurde 1259 während der mongolischen Invasion der Rus zerstört, die Reste werden zwar bis ins 17. Jahrhundert zu Verteidigungszwecken genutzt, doch die Bebauung verlagert sich auf das rechte Pruth-Ufer. Von 1359 bis 1775 gehören die Stadt und ihre Umgebung zum Fürstentum Moldau, hier wird die Stadt in einem Handelsbrief zwischen dem moldauischen Fürsten Alexandru cel Bun und Kaufleuten aus dem damals zu Polen gehörigen Lemberg erstmals urkundlich erwähnt.

1774 wird Czernowitz samt der gesamten Bukowina von Österreich besetzt und 1775 offiziell Teil der Habsburgermonarchie, zunächst als zum Königreich Galizien und Lodomerien gehörig; 1849 wird das Herzogtum Bukowina als eigenes Kronland mit der Landeshauptstadt Czernowitz konstituiert, 1861 das Landesparlament, der Landtag, eingerichtet. In der 1867 gebildeten Doppelmonarchie Österreich-Ungarn gehört das Land bis 1918 zu den im Reichsrat vertretenen Königreichen und Ländern mit deren gemeinsamer kaiserlich-königlicher Regierung in Wien. In diese Zeit fällt eine ambitionierte Kultivierung des Landstriches, wie ferner Czernowitz - zu Beginn des 19. Jahrhunderts eine Stadt von etwas mehr als 5000 Einwohnern - einen steilen Aufstieg erlebt, binnen 80 Jahren verzehnfacht sich die Größe der Stadt, teils Folge eines Ölbooms im Umland, vor allem aber der Erfolg Habsburgs, diesen Land- 
strich auch kulturell aufzurüsten. Wie sehr diese kulturelle Armierung von Bedeutung ist, zeigt sich, als die Stadt Objekt der Rumänisierung wird, berühmt ist das Denkmal, das die lokale Identität in Szene zu setzen versucht, mittels des Bukowiner Ochsen, der den Doppeladler zertrampelt. Zuvor war Kultivierung vorangetrieben worden, wobei die Kultur eine importierte war - 1784 wird erstmals eine künstlerische Aufführung für die seit 1775 präsenten Militärs und Angesiedelten erwähnt -; ebenso ist sie zunächst konservativ-kalmierend, Ion Lihaciu weist auf die frühe Veranstaltung von Bällen hin. (Lihaciu 2006, 67) All das dient der Zivilisierung = Habsburgisierung; wo man damit scheiterte, konnte man noch immer die Interessen der sich widersetzenden Gruppen gemäß dem alten Satz divide et impera gegeneinander ausspielen, also die eingeklagte Pluralität dialektisch wieder für das habsburgische Reich nutzen.

Habsburg und dessen pragmatische Fiktion zeitigen durchaus humane Folgen, freilich indirekt, 1875 wird so von Kaiser Franz Joseph I. anlässlich der 100-jährigen Zugehörigkeit zu Österreich eine Universität mit deutscher Unterrichtssprache gegründet, die Franz-Josephs-Universität Czernowitz, zu der Entscheidung dürfte beigetragen haben, dass 1867 die Universität Lemberg polonisiert worden war. Die Universität umfasst 1893 eine griechisch-orientalisch theologische, eine rechts- und staatswissenschaftliche sowie eine philosophische Fakultät und besaß eine Bibliothek mit 50.000 Bänden, einen botanischen Garten, ein chemisches Labor und ein naturhistorisches Museum. 1892/1893 unterrichten 40 Lehrer 281 Studierende, Czernowitz wird nicht nur wie auch andere Städte im östlichen Habsburgischen Einflussbereich Klein-Wien, sondern ebenso Klein-Heidelberg genannt, mit einem regen Studentenleben: fünf „österreichische“, national indifferente Studentenverbindungen, zwei deutschnationale Burschenschaften, zwei katholisch ausgerichtete Verbindungen, sechs rumänische (fünf davon schlagend), fünf ukrainische (drei davon schlagend), ein polnischer katholischer Verein, eine polnische Burschenschaft, neun jüdische Verbindungen (drei davon schlagend) und acht sonstige Korporationen sind kolportiert, die freilich nicht sämtlich gleichzeitig existieren und teils ineinander über- oder aufgingen - wobei es früh Auseinandersetzungen gibt, insbesondere zu Semesteranfang dürfte es vor allem „für jüdische Schüler und Studenten [...] etwas keineswegs Ungewöhnliches“ gewesen sein, „verprügelt zu werden“ (Menninghaus 1999, 352; Gong 1980, 13).

Daneben steht aber stets ein blühendes Kultur- und Wissenschaftsleben; jedenfalls wird hier ein Fundament gelegt und die Grundlage für die heutige Universität von Czernowitz geschaffen, die allerdings in einem anderen Gebäude - der einstmaligen erzbischöflichen Residenz - ihren Hauptsitz hat und als Dependance der Universität von Iaşi (Rumänien) ins Leben gerufen wurde. Ferner hat Czernowitz damals ein Obergymnasium, eine Oberrealschule, eine Staatsgewerbeschule, eine Lehrerbildungsanstalt und eine landwirtschaftliche Lehranstalt, schließlich ein Landesmuseum. 
Dem Habsburgischen Dispositiv gemäß finden sich hier eine griechisch-orientalische Domkirche (vollendet 1864), eine armenisch-katholische Kirche (1875), eine neue Jesuitenkirche, eine Synagoge und die erwähnte griechisch-orientalische erzbischöfliche Residenz, die Einwohner begründen ebenfalls eine multikulturelle Miniaturmetropole, die sich aus etwa 27.000 Deutschen - sprachenpolitisch wird zu jener Zeit das Jiddische meist hierunter gerechnet -, 10.000 Ruthenen, 8.000 Polen und 8.000 Rumänen konstituiert, wobei das Judentum stark repräsentiert ist, nicht zuletzt wegen des Antisemitismus in vielen nahen Gebieten, rund 17.000 Einwohner sind israelitischen Bekenntnisses; der Umstand, dass Czernowitz bis heute in all diesen Sprachen je seinen Namen hat, bezeugt es: In Czernowitz entstand eine multikulturelle Stadtbevölkerung, wobei der Begriff der Multikulturalität freilich zu differenzieren ist - von der Einschätzung, es habe ein Nebeneinander gegeben, über jene, dass viele Gruppen einander doch unfreundlich gesonnen waren (oder selbst in streitende Untergruppen zerfielen) bis zu jener, dass es tatsächlich eine Multikulturalität und eine entsprechende Kompetenz gegeben habe, reichen hier die Einschätzungen, sicherlich gab es aber eine „Inkongruenzkompensationskompetenz“ (Hainz 2009b, 79), das Wissen und Können, mit den disparaten Kulturen Umgang zu pflegen und dabei einen Sinn für eine gewisse Äquidistanz zu entwickeln. (Stephani 1992, 238) Gewiss gibt es in dieser Multikulturalität zugleich eine Dominanz zunächst der deutschen Sprache, die - der kulturellen Usurpation gemäß - Sprache der ,Leitkultur' ist. Speziell sind die Juden verpflichtet, Deutsch zu lernen, entsprechende Zeugnisse sind Bedingung für Religionspflege wie auch Heirat (vgl. Colin 1994, 17).

Dem kulturellen Aufstieg dieser Landschaft entsprechend floriert auch die Wirtschaft; eine Bierbrauerei, zwei Dampfmühlen, eine Sägemühle, eine Ölfabrik, eine Maschinenfabrik und reger Handel, schon damals insbesondere nach Russland und Rumänien, sind dokumentiert. Zur Stärkung der Infrastruktur sind die guten Bahnverbindungen (als deren Folge man in Czernowitz wiederum bald alle wesentlichen Tageszeitungen vor allem aus Wien beziehen und an den österreichischen und habsburgischen Diskursen partizipieren kann) und die Straßenbahn (später ein Omnibusnetz) erwähnenswert. Bis heute ist Czernowitz ein wichtiger Verkehrsknoten im Südwesten der Ukraine, aufgrund von Straßen- und Eisenbahnstrecken, die Anbindung gen Westen nutzt teils bis heute Habsburgische Strecken.

Czernowitz und mit Einschränkung auch die rurale Bukowina sind bald ein melting pot der Kulturen und Ethnien, von den Czernowitzern heißt es in Karl Emil Franzos’ Roman Der Pojaz, dass sie „doch gewiß Abtrünnige sind“ (Franzos 1979, 125), und zwar verschiedenster Provenienz und aus unterschiedlichen Stereotypen herausgelöst, die allerdings einander mit Vorbehalten begegnen; wird den Russen gerne Brutalität und Verwilderung nachgesagt, „Kosaken haben kein Gesicht“ (Rosenkranz 2001, 56), so den Juden eine übertriebene Kultur - und in den antisemitischen Stereotypen ist das Assimilation -, in der das Selbst verloren gegangen sei: Stehe der Jude krumm, sei er „Bethausjude“, andernfalls stehe er „wie’n Jude in der 
Parade“ (Rosenkranz 2001, 116). Czernowitz bringt Multikulturalität hervor, die fruchtbar wird, aber auch Abgründe, unter die Söhne der Stadt ist ein Stefan Baretzki (1919-1988), SS-Rottenführer im Vernichtungslager Auschwitz, zu rechnen, nicht nur Paul Celan, nicht nur Rose Ausländer. Fraglos verdient freilich das unerhört Humane mehr Beachtung, etwas, das ein babylonisches Gespräch nicht so sehr erzwingt, vielmehr ermöglicht:

Wenn das Herz zum Ausdruck drängte, und miteinander, sprachen die Eltern jüdisch; mit uns Kindern, deutsch; mit der Dienstmagd, dem Gesinde auf der Pachtung und den Einwohnern des Dorfes, ruthenisch; mit den Gutsbesitzern der Umgebung polnisch; und mit den einkehrenden Reisenden, je nach Bedarf, eine dieser Sprachen, deren keine sie wohl musterhaft beherrschen mochten, aber jede gefällig und phantasievoll $\mathrm{zu}$ verwenden schienen, denn es wurde ihnen immer mit Vergnügen und Interesse zugehört. (Rosenkranz 2001, 10)

Die Sprachen, Milieus und Ethnien sind nicht hermetisch voneinander getrennt, man kann wie Rose Ausländers Vater von der Rabbiner-Schule ins völlig säkulare Leben des Geschäftsmanns wechseln, ähnlich ist es mit den Sprachen, Babylon ist für diese Gesellschaft kein Schreckgespenst, sondern Realität, neben den Amtssprachen gibt es noch viele Idiome. Insofern ist Czernowitz natürlich einerseits wie geschildert ein Habsburgisches Projekt, das ein Narrativ unter anderen gebraucht: als erste signifikante Erzählung dessen, was Czernowitz und Kakanien (gewesen) sei; andererseits ist dieses auch wahr: Es gebe und es gab im Vielvölkerstaat Österreich, worin separatistische Ambitionen bestanden, derer man - seien sie nationalistisch motiviert oder bloß Ausdruck des lokalen Establishments, das seine Privilegien zurücksehnte - Herr werden wollte, ein multikulturelles Paradies. Dieses Modell schildert, was es zugleich bewirkt, es ist performativ. Entspricht die Idee von Czernowitz auch nicht überall der Realität, hat sie doch ihre Rechte, und zwar noch in nostalgischen Rückblicken, die davon erzählen, was Czernowitz gewesen sei oder hätte sein können. In dieser Sicht ist das naiv aufgefasst kitschige Wort Celans im Recht, wonach dort „Menschen und Bücher lebten“ (Celan 1986, III/185). Diese Humanisierung ist auch Verklärung; aber opferte man sie dem Recht eines die Habsburg-Ideologie ablösenden postkolonialen Diskurses, machte man sich leicht schuldig, hier mit den Nationalisten die positive Seite des Projekts Habsburg, das Moderne daran, zu diskreditieren, vergessen $\mathrm{zu}$ machen, dass Czernowitz zuletzt Zufluchtsort vor jenen Diskursen war, die man positivistisch bestätigen wollte. Ilana Shmueli schreibt: „[J]eder Czernowitzer hat sein privates, persönliches Czernowitz“ (Shmueli 2006, 95); dieses ist nicht je pauschal zum objektiven Sachverhalt zu stilisieren, aber gegen ein falsch Faktisches zu advozieren. Die Sprachen, die Czernowitz beheimatete, die aber zugleich diese Stadt eigentlich bildeteten, lassen ahnen, dass der homo bukoveniensis (analog zum homo habsburgiensis) eine Konstruktion gewesen sein mag, die wahr wurde. (Hainz 2011, 373-375)

Das, was in Czernowitz beginnt, wird nach dem Ersten Weltkrieg im Zuge der Rumänisierung demontiert. Diese Modernisierung erweist sich als nationalistische 
Aufrüstung, war Czernowitz und hierin insbesondere das Judentum zuvor bedroht die „Czernowitzer Juden konnten nie auf eine allzu freundliche Gesinnung der anderen Stadtbewohner bauen“ (Corbea-Hoișie 1998, 18) -, wird nun programmatisch eine Uniformität umgesetzt, auch sprachenpolitisch, die Czernowitz und hier dem schon zuvor exponierten Judentum zuzusetzen beginnt. Zur Zeit der Rumänisierung beginnt also ein Verfall, literarisch aber der Aufstieg von Czernowitz, das - in vielem ja paradox - erst im fast schon retrospektiven Moment seine größten Dichter zumal der deutsch-jüdischen Literatur hervorbringt; erwähnenwert sind freilich schon zuvor für die rumänische Literatur die Schuljahre Mihai Eminescus (1850-1889) in Czernowitz, ebenso für die deutsche Karl Emil Franzos' (1848-1904) Jugendjahre, die dieser hier verlebte und durch seine Texte über ,Halb-Asien“ diese Region - zu einem Teil der Kultur dieser Region machte. Franzos ist freilich auch durch editorische Leistungen, vor allem seine Verdienste um Georg Büchner, bedeutsam. Literarisch verewigt diese Literaturlandschaft auch Leopold von SacherMasoch mit dem Besuch beim Wunderrabbi von Sadagora, einem Text, der den diesem Verfasser wichtigen topoi folgt, mit Fokus auf dem spirituellen Zentrum Sadagora. Der Czernowitzer Raimund Friedrich Kaindl (1866-1930) macht sich als Historiker und Ethnologe verdient um seine Herkunftsstadt, von der ihn sein Weg nach Graz führt. Wichtig sind schließlich die jiddischen Dichtungen Elieser Steinbargs (1880-1932); neben ihm sind Mosche (Moses) Altman (1890-1981), der mit Steinbarg befreundet aus der Gegend stammt, aber ein bewegtes Leben durchleidet, nämlich nach seiner Rückkehr aus dem Russischen Exil, das ihn vor dem Regime Antonescus bewahrte, als Nationalist (später aber auch als Internationalist) denunziert 1949-1955 in einem Sibirischen Gulag lebt, weiters der viele Male (unter anderem von Rose Ausländer) übersetzte Prinz der jüdischen Ballade Itzig Manger (1901-1969) und als letzter Josef Burg (1912-2009) Proponenten dieser mit Burg ausgestorbenen Literatur.

Die deutsch-jüdische Literatur erlebt aber erst in den Folgejahren, eben jenen des Niedergangs der Stadt, ihr Erblühen. Alfred Margul-Sperber (1898-1967) entstammt Storozynetz aus dem Czernowitzer Umland, er wird der Doyen, der vermittelnd Chancen stiftet und vielen Dichtern in geradezu väterlicher Weise Wege eröffnet. Selbst ein formal konservativer Poet, allerdings von Rang, der freilich moderierend noch in seinen Übersetzungen verfährt, hat er ein Sensorium auch für jene Literatur, die mit Konventionen bricht, etwa jene Paul Celans (1920-1970), den er, als jener emigriert, u.a. Otto Basil empfiehlt (Seng 1998, 58-60); er korrespondiert mit vielen internationalen Größen seiner Zeit, verbindet Czernowitz mit einem literarischen Weltbürgertum und ist zumal indirekt einer, der für die Literatur des 20. Jahrhunderts eminent wichtig war (Motzan 1992, 88; Motzan 2002, 10-12; Hainz 2005, 113-115) Auch seine Erwähnung etwa in Karl Kraus' Fackel bezeugt diese Befunde (Kraus 2000, Bd. 34, 90).

Celan ist retrospektiv das Zentralgestirn der Czernowitzer Literatur, seine literarischen Anfänge spielen mit Versatzstücken eines innigen Expressionismus - gera- 
de auch in den rumänischen Texten seines Frühwerks (Hainz 2006, 217-220) -, doch von Beginn an ist seiner topischen Melancholie eine beißende Ironie beigesellt, die Sprache dekonstruiert, die Tropen und Wendungen ad absurdum führt (Bollack 1998, 127-130; Hainz 2009b, 45; Goßens 2008, 39-45). Celan, oft auf seine dann zudem gern problematisch, nämlich etwa als Versöhnungsgedicht, gedeutete Todesfuge reduziert, hat die Literatur des 20. Jahrhunderts wie wenige geprägt, in der Ahnung von Höhen und Tiefen, aber auch der Ahnung, wann diese vorgespiegelt wurden, Poet und Sprachkritiker, Melancholiker und Vertreter eines maliziösen Humors zugleich. Sicher ist er es, dem Czernowitz nicht zuletzt Beachtung verdankt, wie auch das Narrativ der littérature mineure, wofür freilich vor allem auch Kafkas Prag wesentlich ist. Celan verbindet beim Wort Genommenes so, dass er die Sprache durchdringt, durch sie „hindurch“ (Celan 1986, III/186) ist zu erahnen, was sie sonst unterschlüge.

Zu Margul-Sperbers Schützlingen zählt auch die allerdings kaum jüngere, als Rosalie Scherzer geborene Rose Ausländer (1901-1988), der er etwa bei ihrem ersten Lyrikband Der Regenbogen behilflich war, es dürften auch einige Gedichttitel hier von Margul-Sperber stammen, eine Mentoren-Rolle, die hier Grenzen überschreiten mag (Gutu 2002/03, 69-71); allerdings ist Rose Ausländer eine Lyrikerin, die aufgrund der Emigration während des Ersten Weltkriegs (nach Wien) und dann ihrer USA-Aufenthalte als Dichterin der Bukowina von dieser geprägt und doch atypisch sozialisiert erscheint, vor allem Marianne Moore und E. E. Cummings sowie der amerikanische Kontext in der Zeit ihres Sprachenwechsels (Hainz 2006a) zählen neben den für die Czernowitzer Literatur typischen Einflüsse wie etwa Georg Trakl und Karl Kraus zu der Tradition, der sie sich zurechnet. Zudem ist an ihrer Vita schwerlich zu übersehen, dass sie sich von Einflüssen, obwohl ihr Leben einige Vaterfiguren aufweist, letztlich immer konsequent emanzipierte, sei es der in Czernowitz unter anderem aufgrund des nicht unproblematischen Ethischen Seminars des Denkers - oder Gurus - Friedrich Kettner (Pseudonym von Friedrich Katz, 1886-1957) geradezu populäre Philosoph Constantin Brunner, sei es Margul-Sperber. Czernowitz und die Bukowina hat Ausländer in vielschichtigen Texten bedacht, die zum einen das Narrativ pflegen, das diese Literaturlandschaft in der Tat erblühen ließ, andererseits aber auch Absagen an den Kitsch des Bildes sind. Dieses an Spannungen und Ambivalenz reiche Werk, worin allerdings die Bukowina quantitativ weit weniger Raum einnimmt, als die Rezeption erwarten ließe, bewahrt die Möglichkeit und die Realität dieses Landstrichs, dem die Dichterin, dem Naziterror entronnen, nach Erfahrungen mit dem sowjetischen System endgültig den Rücken zukehrt, wie bis zu einem Grad auch Europa, dessen Frieden sie auch nach der Rückkehr in die deutsche Sprache im Rahmen einer stets provisorischen Existenz in Europa und zuletzt Deutschland, genauer Düsseldorf, nie mehr traut.

Klara Blum (1904-1971) führt ihr Weg gleichfalls in die Diaspora, wobei ihre Existenz letztlich auf einer Lebenslüge gründet, dass nämlich ihr Geliebter, der chinesische Journalist und Regisseur Zhu Xiangcheng, der nach vier Monaten Be- 
ziehung spurlos verschwunden und nach seiner Verhaftung durch die sowjetischen Behörden in einem sibirischen Lager 1943 umgekommen war, lebe und Geheimmissionen nachgehe. Bis 1945 wird Blum die Ausreise aus der Sowjetunion verweigert, danach gelangt sie nach Aufenthalten in Warschau, Prag, Budapest, Bukarest und Paris in die Republik China, 1952 wird sie Professorin für deutsche Sprache und Literatur an der Universität in Nanjing, 1957 an einer Hochschule in Guangzhound, 1954 nimmt die bis zu ihrem Lebensende überzeugte Kommunistin die chinesische Staatsbürgerschaft an und trägt den Namen Zhu Bailan, es erscheinen noch einige deutschsprachige Werke von ihr in der DDR.

Alfred Kittner (1906-1991) ist eine weitere wichtige Stimme dieser Landschaft, ferner ist als Altersgenosse und Freund Celans auch [James] Immanuel Weißglas (1920-1979) zu erwähnen, der mit Celan zu dieser Zeit in regem Austausch stand und im Kontext der Todesfuge von Bedeutung ist (allerdings nicht in jenem Sinne, die man nach Claire Golls Infamien verschiedentlich sehen wollte); sein Werk wird jüngst germanistisch wieder in den Fokus gerückt (Hainz 2010).

Zeitzeugin von Bedeutung ist Edith Silbermann, deren Vater mit seiner Bibliothek ein kulturelles Zentrum der Stadt war. Silbermann, die wie viele andere Czernowitzer zuletzt in Düsseldorf lebte, trug mit ihren Erinnerungen zum facettenreichen Bild der Stadt bei, so auch Stella Avni. Auch Ilana Shmueli, geborene Liane Schindler (1924-2011), hat hierzu sehr Lesenswertes beigetragen, mit akkuraten, uneitlen und reflektierten Memoiren dieser Stadt, von der sie den in der Czernowitzer Memoralistik singulären, zu bedenkenden Satz formuliert, es könnte diese Stadt als Symptom wofür auch immer jedenfalls „vielleicht auch überbewertet“ (Shmueli 2006, 7) sein. Sie, die Ende der 1960er Jahre die letzte Geliebte Celans war, der gemeinsame Briefwechsel ist ein lesenswertes Zeugnis und erschien 2004, und auch über diese Erlebnisse sehr eindrückliche Gedanken hinterließ, porträtiert Zivilisation im Kollaps, gebrochen durch die eigene Sicht, der eines Kindes aus guter Familie also „[w]ir lasen und sprachen hauptsächlich Deutsch und dachten nicht darüber nach, daß es die Sprache des Volkes war, das uns vernichtete“ (Shmueli 2002, 169) -, aber auch durch die Schilderung verschiedener anderer Reaktionen auf diesen Kollaps, so Celans Auffassung, bildungsbürgerlich hierauf zu reagieren sei von einer „Widersinnigkeit“ (Shmueli 2006, 79). Auch der Mehrsprachigkeit widmet sich Shmueli, einem Reichtum, der aber auch Abgründe habe: „Frustrationen, Unsicherheiten, Einengungen. Die vielgerühmte Vielsprachigkeit wurde mir oft zur Sprachlosigkeit. [...] In welcher Sprache liebe ich, fluche ich, träume ich, klage ich und bete ich - in welcher Sprache wird der Tod zu mir kommen?" (Shmueli 2006, 15-16)

Der schon zitierte Alfred Gong (1920-1981) liefert gleichfalls ein Bild, das nichts schönredet und z.B. die Auseinandersetzungen der Ethnien und den Antisemitismus von Czernowitz nicht ausklammert; dies gilt auch von Moses Rosenkranz (1904-2003), einem Dichter von beeindruckender Sprachmelodik und oft beeindruckender Genauigkeit, der gleichwohl im zuweilen zerstrittenen und von Intrige geprägten Chor der Czernowitzer Kultur zweifelsohne auch Fragliches kolportierte. 
Als Zeuge und Sohn Czernowitz' ist auch Georg Drozdowski (1899-1987) anzuführen, der das „Wechselbad von geistiger Weite und fanatischer Verblendung“ (Hagmeyer 2009) in dieser Stadt sehr scharf wahrnahm, ferner Gregor von Rezzori d'Arezzo bzw. Gregor von Rezzori (1914-1998), der durch Geschichten aus Czernowitz und seinem zu Maghrebinien stilisierten Umland in die Literaturhistorie einging, wobei ihm die Rezeption, die vor allem den Anekdoten erzählenden, Stereotypes nicht scheuenden Bonvivant in ihm sehen wollte, Unrecht tat. Es ist unter anderem Jacques Lajarrige sowie dem Czernowitz biographisch, aber auch durch seine Forschungsarbeit innig verbundenen Germanisten Andrei Corbea-Hoişie zu danken, dass Rezzori jüngst eine differenziertere Sichtweise zuteil wurde, die seine Ironie, seinen Sinn für multiple Codierungen und schließlich seinen Realismus würdigt. Kaum ließe sich leugnen, dass Rezzoris Bemerkung, Maghrebinien sei ein Ort, der „im Herzen und in der Seele seiner Menschen“ (Rezzori 2009, 7) liege, wie Celans Blick auf Czernowitz dessen intrikates Verhältnis zum Faktischen beleuchtet, das heute diese Literatur kaum erklären kann: und Czernowitz zurecht als auch heterotopisch versteht. Die Gleichzeitigkeit von Kultur und ihrer Suspension - nicht zuletzt: durch Kulturen - fasst Rezzori bündig so zusammen: Jene Stadt sei „barock in dem Sinne, daß man den unbedingten Glauben an die Notwendigkeit von Formen [...] mit der gleich unbedingten Notwendigkeit, sich darüber lustig zu machen, verbindet.“ (Rezzori 2004, 32) Einem nationalistischen oder chauvinistischen Dispositiv verdankt sich seine Rede von einer Czernowitzer „Sprachzersetzung“ (Rezzori 2004, 286) jedenfalls schwerlich.

Fast alles überstrahlen die wenigen Gedichte der Selma Meerbaum Eisinger (1924-1942), wiewohl sie kaum volljährig im Arbeitslager Michailowka in der Ukraine umkommt. Die mit Paul Celan entfernt Verwandte findet in ihrer Jugend, die durch Lektüren unter anderem Heinrich Heines, Rainer Maria Rilkes, Klabunds und Paul Verlaines geprägt ist, einen ungemein reinen, klaren Stil, der, so sicher er auch ist, sein Bedrohtes bald reflektiert; ihr schmales, aber wichtiges Werk, bestehend aus 57 mit Bleistift niedergeschriebenen Gedichten, entsteht in etwa drei Jahren und verbindet Liebes- und Naturlyrik mit einer fragilen Trauer, die sich retrospektiv wie eine Ahnung ausnimmt. Nicht nur die Dichterin, auch ihr ein Jahr älterer Freund Lejser Fichman, dem die Dichtung gewidmet ist, kommt in der Shoah um, das Album gelangt indes nach Israel.

Man könnte fortfahren, etwa auf die Czernowitzer Vorfahren Albert Drachs (1902-1995) verweisen, aber auch auf Aharon Appelfeld (geb.1932), für den Czernowitz freilich keine prägende Erfahrung mehr ist, oder auf Edgar Hilsenrath (geb. 1926). $\mathrm{Zu}$ ergänzen ist dieses Bild weiters durch die ,anderen' Czernowitzer, wie Klaus Werner schreibt, Walther Rode (1876-1934), ursprünglich Rosenzweig, Verfasser von Texten im Spannungsfeld literarischer und juristischer Diskurse und von „widerstandswilligem moralischen Holz“ (Werner 2001, 174), Wilhelm Reich (1897-1957), Psychiater, Psychoanalytiker, Sexualforscher und Soziologe, den marxistischen Humanisten und Soziologen Maximilien Rubel (1905-1996), und den 
berühmten Biochemiker Erwin Chargaff (1905-2002), der auch als Schriftsteller bedeutsam ist (Werner 2001).

Dokumentiert ist die Breite und Qualität der literarischen Produktion dieser Czernowitzer Jahre durch Anthologien wie Alfred Margul-Sperbers Die Buche, ein Projekt, das der Dichter Ende der 1930er Jahre in Angriff nahm. Der umsichtige Förderer seiner Zeitgenossen stellt eine Auswahl von Texten zusammen, von der lange nur wenig bekannt war, nämlich, wer etwa die Autoren seien, und, dass es einige Konvolute gebe; 2009 wurde sie von Peter Motzan, George Gutu und Stefan Sienerth sorgfältig rekonstruiert und ediert, mit einem einleitenden Editionsbericht, der unter anderem auch die Fragilität von Nachlässen jener Tage erahnen lässt. Anders als die glänzende Zusammenstellung von Klaus Werner, der 1991 das, was von der Bukowina bleiben würde, herauspräparierte und darin eine Ergänzung zum nun präsentierten Band darstellt, ist dieses Panorama zeitnahes Zeugnis. Rose Ausländer ist zunächst am stärksten präsent, bis zu 30 Gedichte wollte der Herausgeber aufnehmen, doch auch weniger bekannte Dichter bekommen eine Stimme, so Salome Mischel mit bis zu 22 Texten. Beide würdigt Margul-Sperber als in der „Frauendichtung“ seiner Zeit maßgeblich. Noch nicht vorgesehen ist zunächst die Aufnahme von Texten des jungen Paul Celan, der erst in der Zusammenstellung B1 vorkommt, dann aber mit 17 Gedichten, während nunmehr die Auswahl aus Ausländers Texten auf ein Drittel gekürzt wurde. Gerahmt mit Margul-Sperbers Der unsichtbare Chor, einem Aufsatz, der die Anliegen expliziert, ist die Kompilation ein Vermächtnis.

Dieser Blüte folgt die Vernichtung, bzw. fällt in diese: Czernowitz wird zunächst brutal rumänisiert, wobei der Antisemitismus insbesondere auffällt, wenngleich nicht in jenem Maße wie in Iaşi und Bukarest, wo der Zulauf der Eisernen Garde noch stärker ist. Systematische Benachteiligung anderer Ethnien als der rumänischen, aber auch Übergriffe, die selbst durch die neue Jurisdiktion nicht gedeckt sind, zerstören das Kulturleben, spätestens „Mitte der 1930er Jahre lösten radikale Kräfte die gemäßigten Führer ab“ (Hausleitner 2005, 65), mitunter damit eine (letztlich hilflose) Radikalisierung anderer Ethnien zeitigend: Die Finanzierung deutscher Kultureinrichtungen durch Deutschland ist etwa rasch dem Einfluss des Nationalsozialismus auf diese förderlich, in „der Czernowitzer Deutschen Tagespost wurde nur Positives über die Entwicklungen im Deutschen Reich berichtet.“ (Hausleitner 2005, 65) Ungünstig wirkt sich zu dieser Zeit ferner der ökonomische Druck der Wirtschaftskrise aus, der der Radikalisierung Vorschub leistet.

Bemühungen um Demokratie und Zusammenleben werden so zum Ringen um Bürgerrechte, doch nur mehr marginalisiert entfaltet sich nun - paradox - das, was man mit Czernowitz und seiner Kultur assoziiert, während bis in die Universitäten eben dies ausgemerzt wird. Das ,Entgegenkommen` Ion Nistors, eben dort Rumänen zu bevorzugen, um die Wogen zu glätten, führt auch hier zur Partikularisierung; das ideologische Programm, „den Juden das Brot aus dem Mund zu nehmen“ (Nistor in Hausleitner 2005, 67), ist für das Klima repräsentativ. Das, was der ethnischen und sozialen Homogenisierung folgt, ist die „schnelle „Säuberung“ (Constantin Loghin 
in Hausleitner 2005, 71), ,Kriegswichtige، (16.500 Juden, weitere 4.500 durch Traian Popovici) entgehen ihr, während Deportationen beginnen. Czernowitz wird, als sich Marschall Ion Antonescu mit Hitler verbündet, vom Faschismus zerstört, zunächst unsystematisch, später planvoll, die Stadt wird in die Kriegswirren gezogen, die schon im Ersten Weltkrieg Spuren hinterlassen hatten. 1943 werden die Juden im deutschen Besatzungsgebiet systematisch ermordet, im rumänischen indes wird der Antisemitismus als Bekämpfung der Partisanen verschleiert betrieben, 1944 sind Nordbukowina und Bessarabien schließlich unter sowjetischer Verwaltung - von erlesener Perfidie ist zu diesem Zeitpunkt Antonescus Schutzbehauptung, die Deportationen, denen bis 1943 61\% der Deportierten zum Opfer gefallen sind, hätten dem Schutz der Juden vor Pogromen gedient (vgl. Hausleitner 2005, 77).

Die die Gefahr ahnen, flüchten, sofern sie können, andere überleben in den Ghettos, oft versteckt; manche kehren wie Rose Ausländer gerade in der Zeit des Faschismus zurück, um Verwandte - in Ausländers Fall die Mutter - zu unterstützen. Viele kommen um. Das kulturelle Leben im Ghetto wird, sofern es sich vollziehen kann, als widersinnig empfunden, ist es doch oft ein (zumindest sprachlich) deutsches Kulturleben. Die Dokumente und Memoiren sind das Leben von Czernowitz nach seinem Untergang, als zuweilen ironisch gebrochene, zuweilen nostalgische Erinnerung lebt es in der Memoralistik derer fort, die es großteils verlassen, zunächst oft nach Bukarest, das für viele nur Zwischenstation wird, für andere wie Margul-Sperber eine provisorische und doch endgültige Heimat. Czernowitz selbst wird der UdSSR einverleibt, später der GUS, es liegt heute in der Ukraine. In Bukarest wird Margul-Sperber zum Kurator seiner Czernowitzer Moderne, die er diplomatisch und moderierend gegen die Vereinfachungen einer Kulturpolitik verteidigt, die unter neuen ideologischen Grundsätzen das, was zunächst als ,jüdisch“ verfolgt wurde, nun der Bourgeoisie zuschlagen will. Die Erfahrungen mit dem NKWD (Vorläufer des KGB) bewegen die als pro-westlich inhaftierte Rose Ausländer zum Exil. Celan bieten Czernowitz und danach ebenso Österreich keine Heimat, er lebt schließlich in Frankreich. Einige Städte - wie New York oder das in diesem Kontext erwähnte Düsseldorf - werden Czernowitzer Enklaven. Czernowitz wird so globalisiert, auf tragische Weise ganz zu jenem „Sprungbrett“ (Rychlo 2005, 10), das es zuvor schon vielen war; heute ist Czernowitz ein topos, der anregend und verführerisch von dem erzählt, was Positivismen und Diskursanalysen - teils zu Unrecht widerlegt $\mathrm{zu}$ haben vermeinen.

\section{Literaturverzeichnis}

Ausländer, Rose: Christian Morgenstern-Translation Kommentiert und hrsg. von Martin A. Hainz. Hamburg: Dr. Kovač, 2012.

Ausländer, Rose: Gesamtwerk in Einzelbänden. Hrsg. von Helmut Braun. Frankfurt a.M.: Fischer Taschenbuch Verlag, 1991-1995. 
Bollack, Jean: „Vor dem Gericht der Toten. Paul Celans Begegnung mit Martin Heidegger und ihre Bedeutung“. Neue Rundschau 109 (1998), H.1, 127-156.

Braun, Helmut: „Ich bin fünftausend Jahre jung“. Rose Ausländer. Zu ihrer Biographie. Stuttgart: Radius, 1999.

Buck, Theo: „Czernowitz als geistige Lebensform“. Maler, Anselm (Hrsg.): Literatur und Regionalität. Frankfurt a.M. u.a.: Peter Lang, 1997, 201-209.

Celan, Paul; Einhorn, Erich: Einhorn. du weißt um die Steine ... Briefwechsel. Hrsg. von Marina Dmitrieva-Einhorn. Berlin Friedenauer Presse, 2001.

Celan, Paul: Gesammelte Werke in fünf Bänden. Hrsg. von Beda Allemann, Stefan Reichert und Rolf Bücher. Frankfurt a.M.: Suhrkamp, 1986.

Chargaff, Erwin: „Die Trennungen“. Daniel, Peter; Diethart, Johannes; Kuhner, Herbert (Hrsg.): Wären die Wände zwischen uns aus Glas / If the Walls Between Us Were Made of Glas Jüdische Lyrik aus Österreich / Austrian Jewish Poetry, Wien: Der Apfel 1992, 142.

Colin, Amy: „Einleitung“. Colin, Amy; Kittner, Alfred (Hrsg.): Versunkene Dichtung der Bukowina. Eine Anthologie deutschsprachiger Lyrik. München: Wilhelm Fink, 1994, 13-24.

Colin, Amy: „Writing from the Margin German-Jewish Women Poets from the Bukovina“. Studies in Twentieth Century Literature Vol. 21, 1 (1997), 9-40.

Corbea-Hoişie, Andrei: „Czernowitz. Bilder einer jüdischen Geschichte“. Andrei Corbea-Hoişie (Hrsg.): Czernowitz. Jüdisches Städtebild. Frankfurt a.M.: Jüdischer Verlag, 1998, 7-26.

Corbea-Hoişie, Andrei: Czernowitzer Geschichten. Über eine städtische Kultur in Mittelosteuropa. Wien u.a.: Böhlau, 2003.

Corbea-Hoişie, Andrei: „Ein Literatenstreit in Czernowitz (1939-1940)“. Études Germaniques Nr 58 (2003) H. 2, 363-377.

Die Buche. Eine Anthologie deutschsprachiger Judendichtung aus der Bukowina. Zusammengestellt von Alfred Margul-Sperber. Aus dem Nachlass hrsg. von George Gutu, Peter Motzan und Stefan Sienerth. München: Institut für Kultur und Geschichte Südosteuropas, 2009.

Emmerich, Wolfgang: Paul Celan. Reinbek b. Hamburg: Rowohlt Taschenbuch Verlag, 1999.

Franzos, Karl Emil: Der Pojaz. Königstein i.Ts.: Athenäum, 1979.

Franzos, Karl Emil: Die Juden von Barnow. Geschichten. Stuttgart, Berlin: Cotta, ${ }^{7} 1905$.

Franzos, Karl Emil: Galizische Erzählungen. Berlin, Weimar: Aufbau, 1980.

Gellhaus, Axel et al.: „Fremde Nähe“. Celan als Übersetzer. Marbach am Neckar: Deutsche Schillergesellschaft, ${ }^{2} 1997$.

Gellhaus, Axel et al.: „Paul Celan. Stationen seines Lebens“. Marbacher Magazin 90/2000, 3-24. Gong, Alfred: Gnadenfrist. Gedichte. Baden bei Wien: Grasl, 1980.

Goßens, Peter: „Das Frühwerk bis zu Der Sand in den Urnen (1938-1950)“. May, Markus; Goßens, Peter; Lehmann, Jürgen (Hrsg.): Celan Handbuch. Leben - Werk - Wirkung. Stuttgart, Weimar: Metzler, 2008, 39-54.

Gutu, George: „Alfred Margul-Sperbers Mentorenrolle für Rose Ausländer und Paul Celan“. Zeitschrift der Germanisten Rumäniens Nr. 11/12, 1-2 (21-24) (2002/03), 69-88.

Gutu, George: Die Lyrik Paul Celans und die rumänische Dichtung der Zwischenkriegszeit. Bukarest: Editura Universitatii din Bucuresti, 1994.

Gutu, George: „Eine Oase rumäniendeutscher Literatur. Der Alfred-Margul-Sperber-Nachlaß in Bukarest“. Florstedt, Renate (Hrsg.): Wortreiche Landschaft. Deutsche Literatur aus Rumänien - Siebenbürgen, Banat, Bukowina. Ein Überblick vom 12. Jahrhundert bis zur Gegenwart. Leipzig: BlickPunktBuch,1998, 201-205.

Hagmeyer, Christa: „Von Czernowitz nach Klagenfurt. Gedichte von Georg Drozdowski in einer Auswahl“. literaturkritik.de, 17.9.2009.

Hainz, Martin A.: „Verhaltenes Ermöglichen - zu Alfred Margul-Sperber (1898-1967)“. Enklaar, Jattie; Ester, Hans; Tax, Evelyne (Hrsg.): Im Schatten der Literaturgeschichte. Autoren, die 
keiner mehr kennt? Plädoyer gegen das Vergessen. Amsterdam, New York: Rodopi, 2005, 113-128.

Hainz, Martin A.: „Das Exil als Verpuppungsstadium - zu Rose Ausländer“. Eidherr, Armin; Langer, Gerhard; Müller, Karl (Hrsg.): Diaspora - Exil als Krisenerfahrung. Jüdische Bilanzen und Perspektiven. Klagenfurt, Wien: Theodor Kramer Gesellschaft, Drava, 2006a, 358-377.

Hainz, Martin A.; Hainz, Daria: „Tauben von Glas. Rumänische Texte aus Celans Frühwerk“. Kutzmutz, Olaf; La Salvia, Adrian (Hrsg.): Halbe Sachen. Wolfenbütteler Übersetzergespräche IV-VI. Erlanger Übersetzerwerkstatt I-II. Wolfenbüttel: Bundesakademie für kulturelle Bildung, 2006b, 217-225.

Hainz, Martin A.: Entgöttertes Leid. Zur Lyrik Rose Ausländers unter Berücksichtigung der Poetologien von Theodor W. Adorno, Peter Szondi und Jacques Derrida. Tübingen: Niemeyer, 2008a.

Hainz, Martin A.: „Ausländer, Rose“. Bautz, Friedrich Wilhelm; Bautz, Traugott (Hrsg.): Biographisch-Bibliographisches Kirchenlexikon Bd. XXIX. Nordhausen: Traugott Bautz, 2008b, Sp. 106-112.

Hainz, Martin A.: Paul Celan. Fadensonnen, -schein und -kreuz. Hamburg: Dr. Kovač, 2009a.

Hainz, Martin A.: „Polylog in statu nascendi. Margul-Sperbers ,Die Buche““. literaturkritik.de Nr. 10, Oktober 2009b.

Hainz, Martin A.: „Tod als Formsache. Zu Immanuel Weißglas“. Corbea-Hoişie, Andrei; Marcu, Grigore; Jordan, Joachim (Hrsg.): Immanuel Weißglas (1920-1979). Studien zum Leben und Werk. Iaşi, Konstanz: Editura Universitatii „Alexandru Ioan Cuza“ Hartung-Gorre, 2010, 273-280.

Hainz, Martin A.: „Es gab inn“. Spiegelungen Nr. 6(60), 4 (2011), 373-377.

Hausleitner, Mariana: „Eine wechselvolle Geschichte. Die Bukowina und die Stadt Czernowitz vom 18. bis zum 20. Jahrhundert“. Braun, Helmut (Hrsg.): Czernowitz. Die Geschichte einer untergegangenen Kulturmetropole. Berlin: Ch. Links, 2005, 31-81.

lorga, Nicolae: „Czernowitz“. Andrei Corbea-Hoişie (Hrsg.): Czernowitz. Jüdisches Städtebild. Frankfurt a.M.: Jüdischer Verlag, 1998, 119-125.

Kraus, Karl: Die Fackel Nr. 1 (1899) - Nr. 922 (1936). Hrsg. von Friedrich Pfäfflin. München: Saur, 2002.

Lihaciu, Ion: „Aufblühendes kulturelles Leben im alten Czernowitz. Zum kreativen Milieu einer Provinzmetropole“. Spiegelungen Nr. 1, 1.(2006), 67-73.

Lihaciu, Ion: Czernowitz 1848-1918. Das kulturelle Leben einer Provinzmetropole. Kaiserslautern, Mehlingen: Parthenon, 2012.

Margul-Sperber, Alfred: Geheimnis und Verzicht. Das lyrische Werk in Auswahl. Hrsg. von Alfred Kittner. Bukarest: Kriterion, 1975.

Margul-Sperber, Alfred: Ins Leere gesprochen. Ausgewählte Gedichte 1914-1966. Hrsg. von Peter Motzan. Aachen: Rimbaud, 2002.

Meerbaum-Eisinger, Selma: Ich bin in Sehnsucht eingehüllt. Gedichte eine jüdischen Mädchens an seinen Freund. Hrsg. von Jürgen Serke. Frankfurt a.M.: Fischer Taschenbuch Verlag, 1994.

Menninghaus, Winfried: „,Czernowitz / Bukowina“ als literarischer Topos deutsch-jüdischer Geschichte und Literatur“. Merkur 600 (1999), 345-357.

Mittelmann, Hermann: Illustrierter Führer durch die Bukowina (1907/1908). Neu hrsg. von Helmut Kusdat. Wien: Mandelbaum, 2001, ${ }^{2} 2002$.

Motzan, Peter: „Alfred Margul-Sperber (1898-1967). Eine Porträtskizze“. Corbea-Hoişie, Andrei; Gutu, George; Hainz, Martin A. (Hrsg.): Stundenwechsel. Neue Lektüren zu Rose Ausländer, Paul Celan, Alfred Margul-Sperber und Immanuel Weißglas. Konstanz: Hartung-Gorre Verlag, 2002, 10-42.

Motzan, Peter: „Der Lyriker Alfred Margul-Sperber. Ein Forschungsbericht nebst einer kurzen Nachrede“. Corbea, Andrei; Astner, Michael (Hrsg.): Kulturlandschaft Bukowina. Studien zur 
deutschsprachigen Literatur des Buchenlandes nach 1918. Konstanz: Hartung-Gorre, 1992, 88-101.

Neumann, Hans: „Zurück in die Gegenwart. Czernowitzer Lebensart in der memoralistischen Prosa Gregor von Rezzoris und Georg Drozdowski“. Bauer, Markus (Hrsg.): Zum Thema Mitteleuropa. Sprache und Literatur im Kontext. Konstanz: Hartung-Gorre Verlag, 2000, 165-188.

Rezzori, Gregor von: Blumen im Schnee. Portraitstudien zu einer Autobiographie, die ich nie schreiben werde, auch: Versuch der Erzählweise eines gleicherweise nie geschriebenen Bildungsromans. Aachen: Rimbaud, 2004.

Rezzori, Gregor von: Ein Hermelin in Tschernopol. Ein maghrebinischer Roman. Hrsg. von Gerhard Köpf, Heinz Schumacher und Tilman Spengler. Berlin: Berliner Taschenbuch Verlag, 2004.

Rezzori, Gregor von: Maghrebinische Geschichten. Reinbek bei Hamburg: Rowohlt Taschenbuch Verlag, 2009.

Rosenkranz, Moses: Kindheit. Fragment einer Autobiographie. Hrsg. von George Gutu und Doris Rosenkranz. Aachen: Rimbaud, 2001.

Rychlo, Peter: „Czernowitz als geistige Lebensform“. Braun, Helmut (Hrsg.): Czernowitz. Die Geschichte einer untergegangenen Kulturmetropole. Berlin: Ch. Links, 2005, 7-29.

Seng, Joachim: Auf den Kreis-Wegen der Dichtung. Zyklische Komposition bei Paul Celan in den Gedichtbänden bis „Sprachgitter“. Heidelberg: Winter, 1998.

Shmueli, Ilana: Ein Kind aus guter Familie. Czernowitz 1924-1944. Aachen: Rimbaud, 2006.

Shmueli, Ilana: „Über mein Czernowitz erzählen (1924-1944)“. Gordon, Cécile; Kusdat, Helmut (Hrsg.): An der Zeiten Ränder. Czernowitz und die Bukowina. Geschichte - Literatur Verfolgung - Exil. Wien: Theodor Kramer Gesellschaft, 2002, 161-172.

Sienerth, Stefan: „Alfred Margul-Sperbers Korrespondenz mit siebenbürgisch-sächsischen Autoren“. Corbea-Hoişie, Andrei; Gutu, George; Hainz, Martin A. (Hrsg.): Stundenwechsel. Neue Lektüren zu Rose Ausländer, Paul Celan, Alfred Margul-Sperber und Immanuel Weißglas. Konstanz: Hartung-Gorre Verlag, 2002, 79-93.

Sienerth, Stefan: „Rosenkranz, Moses“. Neue Deutsche Biographie 22 (2005), 71-72.

Stephani, Claus: „Zum jüdischen Handwerk in der Bukowina. Anmerkungen am Rande einer Statistik“. Corbea, Andrei; Astner, Michael (Hrsg.): Kulturlandschaft Bukowina. Studien zur deutschsprachigen Literatur des Buchenlandes nach 1918. Konstanz: Hartung-Gorre, 1992, 237-242.

Stiehler, Heinrich: „Der junge Celan und die Sprachen der Bukowina und Rumänien“. Gordon, Cécile; Kusdat, Helmut (Hrsg.): An der Zeiten Ränder. Czernowitz und die Bukowina. Geschichte - Literatur - Verfolgung - Exil. Wien: Theodor Kramer Gesellschaft, 2002, 115-128.

Stiehler, Heinrich: „Die Zeit der Todesfuge. Zu den Anfängen Paul Celans“. Akzente 1 (1972), 11-40. Weißglas, Immanuel: Aschenzeit. Gesammelte Gedichte. Aachen: Rimbaud, 1994.

Werner, Klaus: „Die ,anderen“ Czernowitzer Abkömmlinge deutsch-jüdischer Ideengeschichte und Literatur. Walther Rode, Wilhelm Reich, Maximilien Rubel und Erwin Chargaff“. Aschkenas 11 (2001), H.1, 161-214.

Werner, Klaus: Erfahrungsgeschichte und Zeugenschaft. Studien zur deutsch-jüdischen Literatur aus Galizien und der Bukowina. München: Institut für deutsche Kultur und Geschichte Südosteuropas, 2003.

Werner, Klaus: „Noch einmal. Zum ,Wunder‘ von Alfred Margul-Sperbers Zwischenkriegslyrik. Ein Diskussionsbeitrag“. Corbea-Hoişie, Andrei; Gutu, George; Hainz, Martin A. (Hrsg.): Stundenwechsel. Neue Lektüren zu Rose Ausländer, Paul Celan, Alfred Margul-Sperber und Immanuel Weißglas. Konstanz: Hartung-Gorre Verlag, 2002, 43-56.

Winkler, Markus: „Immanuel Weißglas (1920-1979)“. Spiegelungen Nr. 6(60), 4 (2011), 403-404. 